Sitzungsberichte

der Akademie der Wissenschaften der DDR

\title{
Herbert Hörz
}

Philosophie

und Ökologie 
